\title{
Analysis of deep sequencing Exosome-microRNA expression profile from Chicken Type $₫$ Pneumocytes derived reveals potential role of gga- miRNA-451 in inflammation
}

\author{
Yabo Zhao \\ Huazhong Agriculture University \\ Yali Fu \\ Huazhong Agriculture University \\ Mengyun Zou \\ Huazhong Agriculture University \\ Yingfei Sun \\ Huazhong Agriculture University

\section{Xun Yin} \\ Huazhong Agriculture University \\ Lumeng Niu \\ Huazhong Agriculture University \\ Xiuli Peng ( $\nabla$ xiulipeng918@163.com ) \\ Huazhong Agriculture University
}

\section{Research}

Keywords: Exosomes, gga-miR-451, YWHAZ, Mycoplasma gallisepticum (HS strain), inflammatory cytokines

Posted Date: November 20th, 2019

DOl: https://doi.org/10.21203/rs.2.17460/v1

License: (c) (1) This work is licensed under a Creative Commons Attribution 4.0 International License. Read Full License 


\section{Abstract}

Background: Exosomes are nanosized extracellular vesicles secreted by multiple cells in the body, including those located in the respiratory tract and lungs. They are emerging as important inflammatory mediators and can release their contents, especially microRNAs (miRNAs), to both neighboring and distal cells. Mycoplasma gallisepticum (MG) can target host cell and cause chronic respiratory disease (CRD) in chickens. Although exosomal miRNAs have been demonstrated to produce an important effect on microbial pathogenesis and inflammatory response as crucial regulatory noncoding RNAs, the mechanism by which exosomal miRNAs regulate MG-induced inflammation remains to be elucidated.

Methods: the expression of exosome-microRNA derived from MG-infected chicken type $₫$ pneumocytes (CP- $₫)$ was screened, and the target genes and function of differentially expressed miRNAs (DEGs) were predicted. To verify the inflammatory functions of exosomal gga-miR-451 via targeting YWHAZ, Western blot, ELISA, and RT-qPCR were used in this study.

Results: A total of 722 miRNAs were identified from the two exosomal small RNA (sRNA) libraries, and 279 novel miRNAs were discovered; 30 miRNAs (9 up-regulated and 21 down-regulated) were significantly changed (Pख0.05). Function annotation analysis of DEGs showed that the target miRNAs were significantly enriched in treatment group, such as cell cycle, Toll-like receptor signaling pathway and MAPK signaling pathway, etc. The results have also confirmed that gga-miR-451-absent exosomes derived from MG-infected CP- $\triangle$ cells increased inflammatory cytokine production in DF-1 (chicken embryo fibroblast) cells, and Wild Type CP-囚 cells-derived-exosomes displayed protective effects.

Conclusion: our work suggests that exosomes from MG-infected CP- $₫$ cells alter the dynamics of the DF-1 cells, and may contribute to pathology of the MG infection via exosomal gga-miR-451 targeting YWHAZ involving in inflammation. This could potentially be used as a biomarker for diagnostics and treatment.

\section{Background}

Mycoplasma galisepticum (MG) is one of the most important avian pathogens. It is established that MG infection causes the chronic respiratory disease (CRD) leading to respiratory symptoms including rales, coughs, ocular discharge, and nasal discharge in chickens and turkeys $(1,2)$. Increased embryonic mortality, reduced weight gain, and egg production have been found in infected chickens, typically causing considerable economic losses to the poultry industry $(3,4)$.

miRNA is one of the short endogenous single non-coding RNA of approximately 18-26 nucleotides (nt) in length, which exists in eukaryotic cells (5). Mature miRNA is produced by shear processing of a series of nucleases from the longer primary transcripts, and target mRNA via the complementary pairing of bases to regulate the target mRNA expression or inhibit the translation of the target mRNA according to the degree of bases complementarities (6). Previous studies have confirmed that miRNAs play an important role in biological functions such as apoptosis, cell cycle, metabolism, cell differentiation or development, etc. $(5,7)$. Recent studies have shown that miRNAs are involved in the occurrence and development of 
inflammatory diseases (8). Our previous studies have revealed that miRNAs are associated with MG infection, and that they were significantly changed in chicken lungs at 3 day post-infection with 36 downregulated and 9 up-regulated miRNAs belonging to 31 miRNA families (9). Our laboratory has reported that the gga-miR-16-5p was upregulated in MG-infected fibroblast cells (DF-1), and gga-miR-146c represses the expression of MMP16, activating TLR6/MyD88/NF-KB pathway for promoting cell proliferation to defend against MG infection $(10,11)$. The mature sequences of miR-451 contain $22 \mathrm{nt}$ in length and are highly conserved pending evolvement (12). Recent data clarified the role of miR-451 in the cell apoptosis, migration, and invasion. miR -451 has also been reported to play a critical role in regulating a variety of human and animal diseases, such as influenza, cancer, cerebral ischemia/reperfusion injury, and vascular endothelial dysfunction (13-15). It is worth mentioning that gga-miR-451 regulates the MG-induced production of inflammatory cytokines via targeting YWHAZ (16). Moreover, our previous study also confirmed that AhR:Arnt bind to the gga-miR-451 promoter and facilitate gga-miR-451 transcription in DF-1 cells (17).

Exosomes are the small membranous vesicles $(30-150 \mathrm{~nm}$ ) released by many kinds of cells (including $B$ lymphocytes, endothelial cells, epithelial cells) and body fluids. They are known to represent the characteristics of the cells which secrete them $(18,19)$. The mode of intercellular communication which had gained positive acknowledgement is mediated by exosomes. In juxtracrine or ectodomain cleavagebased signaling, exosomes can regulate the non-selective transfer of exosomal proteins and/or RNA to the target cell via fusing with the target cell (19). In addition to cellular proteins, lipids and functional RNA molecules and other non-coding RNAs (miRNAs and IncRNAs) were also found in exosomes. Growing evidence indicates that miRNAs that are transferred by exosomes are also biologically active via regulating the mRNA level in target cells after entering the target cells $(20,21)$. Exosomes-miRNAs secreted from the cell to the extracellular environment can modulate gene expression and cell function, playing a crucial role in several processes such as inflammation and immune response $(22,23)$. Previously, we reported that several miRNAs play a key role in inflammatory processes induced by MG. However, much of our understanding regarding miRNAs mediated effects on mechanisms of MG infection is based on the use of 'unpackaged' miRNAs, which regulate the original cells and tissues. The function of exosomal miRNAs derived from MG infected-chicken lung epithelial cells on both neighboring and distal cells are still unknown. To expand on our knowledge, it is now critical to assess the effect of miRNAs in a biologically relevant setting in which they are present as exosomal cargo. The purpose of this study is to screen and annotate the differentially expressed exosomal miRNAs in MG infected-CP- $\mathbb{Z}$ cells, and to evaluate the function and potential mechanism of exosomal gga-miR-451 in DF-1 cells, which provides a valuable miR-451 as diagnostic markers and therapeutic targets in MG infected chickens.

\section{Materials And Methods}

2.1 Reagents 
The primary antibody anti-YWHAZ was obtained from Sangon Biotech (Cat. D155211-0025, Shanghai, China), and the primary anti-CD9 and anti-CD63 were purchased from Abcam (Cat.ab92726 and ab193349, Shanghai, China), the primary antibody against glyceraldehyde-3-phosphate dehydrogenase (GAPDH) was acquired from Proteintech (Cat.60004-1-1g, Wuhan, China). The secondary antibody of horseradish peroxidase-conjugated anti-mouse/rabbit was purchased from Sangon Biotech (Shanghai, China). The oligonucleotides of gga-miR-451 mimics and Negative Control (double-stranded chemically modified oligonucleotides) were synthesized by GenePharma (Shanghai, China). The sequences of all the primers and the sequences of RNA oligonucleotides used in the study are shown in Table S1 and Table S2, respectively.

\subsection{CP-II Isolation and DF-1 Cells Culture}

$\mathrm{CP}-\triangle$ cells were isolated as described previously (24), with modifications. Briefly, lungs were removed from 14-day-old of White Leghorn specific-pathogen-free (SPF) chicken embryos and were cut into very small tissue blocks. Then $0.25 \%$ trypsin and $0.1 \%$ IV collagenase (Invitrogen-Gibco, Carlsbad, CA, USA) were used for digestion at $37^{\circ} \mathrm{C}$ for $10 \mathrm{~min}$ and $15 \mathrm{~min}$, respectively. Cell suspensions were filtrated by a 200mesh sieve, resuspended with $10 \%$ fetal bovine serum (FBS; Invitrogen, Carlsbad, CA, USA) in a 150-mm sterile culture plate, and incubated for $1 \mathrm{~h}$. Supernatants with the unattached cells were then collected three times. The unattached cells were centrifuged at $1200 \mathrm{r} / \mathrm{min}$ for $5 \mathrm{~min}$, re-suspended in fresh Dulbecco's modified Eagle's medium (DMEM) for three times, and filtrated by 400 mesh sieves. After the cell count was completed, cells were with $20 \% \mathrm{FBS}$ and concentration was adjusted to $2 \times 10^{6} / \mathrm{ml}$ and then inoculated into $150-\mathrm{mm}$ sterile culture plate. Cells were incubated for $18 \mathrm{~h}$ at $39^{\circ} \mathrm{C}$. The attaching cells on culture dish were $\mathrm{CP}-\mathbb{\Downarrow}$ cells. Invert microscope and transmission electron microscope (TEM) were used to identify the cells.

The chicken embryonic fibroblast cell line (DF-1), obtained from ATCC (Manassas, VA, USA), were maintained at $39{ }^{\circ} \mathrm{C}$ in a $5 \% \mathrm{CO}_{2}$ atmosphere in DMEM (Invitrogen, Carlsbad, CA, USA) mixed with $1 \%$ penicillin-streptavidin-glutamine (PSG, Invitrogen, USA) and $10 \%$ fetal bovine serum (FBS, Invitrogen, USA).

\subsection{Mycoplasma strains}

MG-HS, a virulent strain, was isolated from a chicken farm in Hubei, China and conserved in the State Key Laboratory of Agricultural Microbiology, Huazhong Agricultural University (Wuhan, China) $(25,26)$. The MG-HS strain was cultured, and the concentration was determined as previously described. The viable number of MG-HS in suspension was measured using a color-changing unit (CCU) assay (27). 


\subsection{Infection Experiments}

When CP- $\nabla$ cells in the experimental group reached $80-90 \%$ confluence, twelve $150 \mathrm{~mm}$ plates of the cells were incubated in medium without antibiotics. Six plates of the cells were infected with $2 \mathrm{ml} / \mathrm{plate}$ of MG$\mathrm{HS}$ at the mid-exponential phase $\left(1 \times 10^{12} \mathrm{CCU} / \mathrm{ml}\right)$, whilst the other six plates of the cells uninfected with MG-HS used as a control. At $12 \mathrm{~h}$ post-infection, the medium of the cells in both groups was changed to exosome-depleted media prepared by ultracentrifugation of FBS for $70 \mathrm{~min}$ at $100,000 \times \mathrm{gg}(28)$. The culture supernatant and the cells were collected at 12-60 hpi for further experiments.

\subsection{Exosome isolation and characterization}

The CP- $\rrbracket$ cells culture supernatants in both groups were collected and immediately filtered through a 0.22$\mathrm{mm}$ filter (Millipore, USA) and centrifuged within $30 \mathrm{~min}$ at $2000 \times \mathrm{g}$ for $10 \mathrm{~min}$ at $4{ }^{\circ} \mathrm{C}$. Cell culture exosomes were isolated and characterized as described previously (29-31). Briefly, the supernatant was collected and then centrifuged at $10,000 \mathrm{~g}$ for $40 \mathrm{~min}$ at $4{ }^{\circ} \mathrm{C}$. The resulting supernatant was transferred to a new ultracentrifuge tube and centrifuged at $100,000 \times \mathrm{g}$ for $2 \mathrm{~h}$ at $4^{\circ} \mathrm{C}$ (Beckman Optima XE-90, SW32 Ti rotor). The supernatant was aspirated and the pellet was suspended in pure PBS (HyClone, USA) and centrifuged at $100,000 \times \mathrm{g}$ for another $2 \mathrm{~h}$ at $4{ }^{\circ} \mathrm{C}$. The purified exosomes were resuspended in 50 $\mathrm{LL}$ PBS and used for experimental procedures or stored at $-80^{\circ} \mathrm{C}$. For nanoparticle tracking analysis (NTA), exosomes were diluted with PBS over a range of concentrations to obtain between 10 and 100 particles per image before analysis. The ZetaView Nanoparticle Tracking Analyzer (Particle Metrix, Germany) was used to automatically measure the average diameter and concentration. For Transmission electron microscopy, a 10- $\mu \mathrm{L}$ aliquot of the suspended exosomes was applied to a carboncoated copper grid. Then the sample was negatively stained with $2 \%$ uranyl acetate after drying. Micrographs were obtained under a HITACHI H-7650 transmission electron microscope (HITACHI, Japan).

\subsection{Exosome labeling}

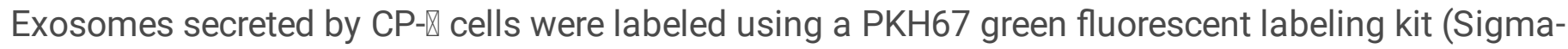
Aldrich, MINI67, MO, USA) to examine the uptake of exosomes by DF-1 cells in vitro. Labeled exosomes were incubated with DF- 1 cells at $39^{\circ} \mathrm{C}$ for $6 \mathrm{~h}$ and then fixed. Fluorescent images were taken with a Confocal Laser Scanning Microscope (ZEISS LSM 800 META, Carl Zeiss Imaging, Germany).

\subsection{Exosome sRNA sequencing and data processing}

Exosomes were isolated from samples and characterized as described above. Total RNA from exosomes in both groups $(n=3)$ was used for sRNA sequencing. Library preparation and sRNA sequencing was 
performed by Ribobio (Guangzhou, China). In brief, total RNA samples were fractionated and only small RNAs ranging from 18 to 40 nts were used for library preparation. After amplification by 15 -cycle PCR, the libraries were sequenced using the Illumina $\mathrm{HiSeq}^{\mathrm{TM}} 2500$ platform. Clean reads were collected from raw reads by removing the adapter dimers, glow quality, and contaminated reads. Then, clean reads of sRNA were mapped to a reference sequence by BWA 0.7.12 (32). Mapped sRNA tags were used to look for known sRNA, and miRbase version 21.0 (www.mirbase.org), Rfam12.1 (rfam.xfam.org), and Pirnabank (pirnabank.ibab.ac.in) were used for reference.

\section{8 miRNA target prediction and function annotation}

Prediction of target genes of differentially expressed miRNAs ( $\left|\log _{2}{ }^{(\text {Fold Change) }}\right| \geq 1$, P-value $\left.\otimes 0.05\right)$ was performed by RNAhybrid, miRanda, and PITA. GOseq based Wallenius non-central hyper-geometric distribution was performed for Gene Ontology (GO) enrichment analysis. KOBAS (v2.0) software was performed for Kyoto Encyclopedia of Genes and Genomes (KEGG) pathway analysis and the corrected pvalue (FDR) cut-off was set at $0.05(33,34)$.

\subsection{Real-time quantitative PCR}

The extraction of total RNA from exosomes and reverse-transcribed into CDNA were performed using a themiRcute Plus miRNA First-Strand cDNA Synthesis Kit (\#KR211-02, Tiangen Biotech Co., Ltd, Beijing, China), according to the manufacturer's recommendation. The miRNA RT-qPCR analysis was performed using a miRcute Plus miRNA qPCR Detection Kit (SYBR Green) (\#FP411-02, Tiangen Biotech Co., Ltd, Beijing, China) and a Bio-Rad CFX384 TouchTM instrument (Bio-Rad, USA). Total RNA from cells was isolated using the TRIzol reagent according to the manufacturer's instructions (Invitrogen, Carlsbad, CA, USA), and cDNA was synthesized using the Prime Script ${ }^{\mathrm{TM}} \mathrm{RT}$ reagent kit with gDNA Eraser (TaKaRa, Japan). The RT- PCR was subsequently determined using a Bio- TransStart Top Green qPCR SuperMix (TRANSGEN, China). RT-qPCR was performed to measure the expression levels of miRNAs and YWHAZ with chicken 5S-RNA and GAPDH mRNA used as internal controls. Data was analyzed using the $2^{-D D C t}$ method. The primers of miRNA, mRNA and 5s-RNA are presented in Table S1.

\subsection{Western blotting}

The total proteins were extracted from exosomes and DF-1 cells at $48 \mathrm{~h}$ post-transfection or exosometreated, and their concentrations were then determined using a BCA Protein Assay Kit (TRANSGEN, China). Equal amounts of protein ( $5 \mu \mathrm{g}$ of exosomal proteins, $50 \mu \mathrm{g}$ of cells proteins) were transferred to nitrocellulose membranes after separation using $12 \%$ SDS-polyacrylamide gel electrophoresis (SDS- 
PAGE) (Beyotime, China) and blocked in $5 \%(\mathrm{w} / \mathrm{v})$ skimmed milk at $25 \pm 1^{\circ} \mathrm{C}$ for $1 \mathrm{~h}$, followed by incubation overnight at $4^{\circ} \mathrm{C}$ with the previous antibodies (anti-CD9, CD63, YWHAZ, or GAPDH). After washing with TBST, the membranes were incubated with the secondary antibodies at room temperature for $1.5 \mathrm{~h}$. After another wash with TBST, the protein expressions on the membranes were detected using an $\mathrm{ECL}^{\mathrm{TM}}$ detection system (Bio-Rad, USA).

\subsection{ELISA}

After exosome-treated or transfection with siRNA in 6-well plates, the DF-1 cells were incubated for $36 \mathrm{~h}$, then the culture supernatants were collected from the treated cells for determination of the inflammatory factor levels, including TNF- $\alpha$ and IL-1 $\beta$. using. All experiments were performed according to the manufacturers' protocols of the enzyme-linked immunosorbent assay (ELISA) kits (Bio-Swamp, China).

\subsection{Statistical analysis}

Data were presented as the mean \pm SEM and analyzed by SPSS 20.0 software for statistical analysis. ANOVA or Student's $t$-test was used to determine significant differences between groups. A value of $p<$ 0.05 was considered statistically significant and $p<0.01$ considered extremely significant. (* $P \otimes 0.05$, ** $P]$ 0.01; different lowercase letters represent $P<0.01$ ).

\section{Results}

3.1 CP- $₫$ cells-derived exosomes were isolated and identified in morphology and phenotype.

The $\mathrm{CP}-\mathbb{X}$ cells shaped monolayers when adherent cultured for $18 \mathrm{~h}$, were small and cycloid, grouped together to form islands (Fig. 1A). The morphology of CP- $\$ cells was observed by optical microscope and transmission electron microscopy (TEM). The cells have one or more osmophilic lamellar bodies and the surface microvilli (Fig. 1B). The exosomes were isolated from the media and collected from MG-infected

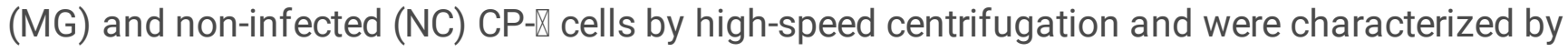
nanoparticle tracking analysis (NTA) and transmission electron microscope (TEM). These results showed an average particle diameter of 30-150 nm (Fig. 1C), double layer membrane structure of round-shaped vesicles, and diameters about 100nm (Fig. 1D). The exosomal surface markers (cluster of differentiation 9 (CD9) and CD63) could be detected using western blotting (Fig. 1E). There was difference in the MGinfected and non-infected CP- $\triangle$ cells, the group of MG-Infection is more consistent with previous reports on exosomes (35). These results demonstrate that CP- $\triangle$ cells-derived particles collected in this experiment were identified as exosomes. 


\subsection{Sequence analysis}

To identify exosomal miRNAs and their expression levels from MG-infected and non-infected CP- $\nabla$ cells, two small RNA libraries were constructed for deep sequencing. The total reads reached more than thirteen million, and approximately $90.16 \%$ clean reads remained in Table S3. These clean reads were matched to authoritative miRNA/rRNA/tRNA/snRNA/snoRNA databases, such as miRbase version 21, Rfam12.1, and Pirnabank. The length distribution range of these libraries is $19-24 \mathrm{nt}$, and $22 \mathrm{nt}$ is the main length in the miRNAs sequence distribution.

\subsection{Differentially expressed miRNAs}

A total of 722 mature chicken miRNAs were identified from the two exosomal sRNA libraries, and 279 novel miRNAs were discovered using miRDeep2 software (Table S4). As shown in Fig. 2A, the differential enriched miRNA profiles of all samples of MG-infected (MG) and non-infected (NC) were evaluated by DESeq. The transcriptional level of co-expressing miRNAs was compared between MG-infected and noninfected libraries (Fig. 2B). There were 469 of 722 miRNAs (65\%) were equally-expressed in the two libraries, while 253 miRNAs (35\%) were differential expressed with a fold-change $>2$ ( $\mid \log _{2}($ Fold Change) $\mid \geq 1)$. Among these differential expressed miRNAs, only 30 miRNAs ( 9 up-regulated and 21 down-regulated) were significantly changed (P-value冈0.05) and 17 miRNAs (3 up-regulated and 14 down-regulated) were significantly changed (P-value区0.01) in the MG-infected vs. non-infected groups (Fig. 2C). The details of the known chicken miRNAs and their log2 (fold-change) and P-value were shown in Table 1. and then applied to evaluation.

\subsection{Functional annotation and enrichment analysis of predicted target genes of the differentially expressed miRNAs}

To further understand of the biological function of miRNAs in CP- $₫$ cells-derived exosomes, a target prediction of miRNAs with $\log _{2}{ }^{\text {(fold-change) }} \geq 1$ and P-value $\otimes 0.05$ was performed using miRanda, Pita, and RNAhybrid. Gene Ontology (GO) functional annotation of target genes was conducted with assignments to biological process (BP), cellular component (CC) and molecular function (MF) categories. The results showed that some items of BP were involved in regulating cellular process, single-organism process, and metabolic process, some items of $\mathrm{CC}$ were involved in regulating cell part, intracellular, and intracellular membrane-bounded organelle, and some of items of MF were involved in regulating protein binding, organic cyclic compound binding, and catalytic activity (Fig. 3; corrected p-value 0.05 ). Analysis of Kyoto Encyclopedia of Genes and Genomes (KEGG) pathway for these target genes was performed $(p<0.05)$. These target genes were mainly enriched in the following pathways: apoptosis, cell cycle, focal adhesion, tight junction, influenza A, Toll-like receptor signaling pathway, Wnt signaling pathway, Cytokine-cytokine receptor interaction and MAPK signaling pathway (Fig. 4). These pathways reflect the physiological processes of MG-infection and the potential regulatory mechanisms underlying them. 


\subsection{Exosomal miRNAs interaction network for elements involved in cell cycle, apoptosis, and Toll-like receptor signals}

Our previous studies have shown that cell cycle, apoptosis, and Toll-like receptor signaling pathways are activated and play an important role in the inflammatory response to MG infection $(16,36)$. We found that 20 predicted targets of DEGs are involved in regulating apoptosis, 16 predicted targets of DEGs are involved in cell cycle, and 16 predicted targets of DEGs are involved in Toll-like receptor signaling pathway (Fig. 5). Interestingly, many miRNAs (21 of 30) negatively regulate these three pathways by targeting the positive genes. Moreover, some other pathways are also modulated by DEGs, such as mitogen-activated protein kinase (MAPK) and Cytokine-cytokine receptor interaction signals.

\subsection{RT-qPCR verification}

To validate the reliability of the sequencing results, a total of 8 differentially expressed exosomal miRNAs were selected for the Poly (A) Plus real-time PCR. RT-qPCR results showed that the expression of gga-let7d, gga-miR-451, gga-miR-133-3p and gga-miR-223 were significantly downregulated in MG-infected group compared to control groups. gga-miR-193a-3p and gga-miR-33-5p were up-regulated in MGinfected group as compared with the control groups. In contrast, gga-miR-460b-5p and gga-miR-202-5p had no significant difference (Fig. 6). These results indicated that exosomes derived from MG-infected $\mathrm{CP}-\mathbb{\nabla}$ cells in early infection may play a significant role in the immune inflammatory process. Consistent with this hypothesis, gga-miR-451 is reportedly involved in the immune inflammatory process (16), suggesting that gga-miR-451 may play a crucial role in immune regulation during early infection.

\section{7 gga-miR-451-desent exosomes derived from MG-infected CP- $\$ cells increases inflammatory cytokine production in DF-1 cells}

Previous data showed that gga-miR-451 expression is significantly elevated during MG infection in DF-1 cells. To verify this result in chicken lung epithelial cells, CP- $\triangle$ cells were infected with MG-HS. At $48 \mathrm{~h}$ post-infection, RT-qPCR result showed that gga-miR-451 level was significantly increased in the CP- $₫$ cells (Fig. 7A). Interestingly, gga-miR-451 level is significantly decreased in the exosomes derived from MG-

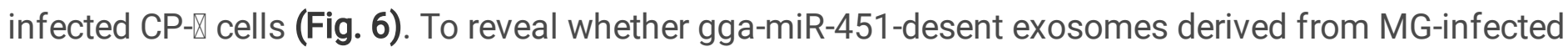
$\mathrm{CP}-\mathbb{\nabla}$ cells are involved in the regulation of inflammation to commute between lung epithelial cells and

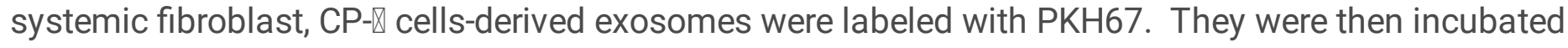
with DF- 1 cells for $6 \mathrm{~h}$ at $37^{\circ} \mathrm{C}$, and observed by confocal microscopy, which revealed the incorporation of exosomes into DF-1 cells (Fig. 7B). Next, DF-1 cells were incubated with exosome derived from MG-

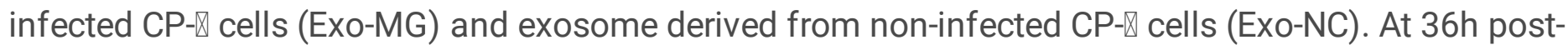
treated, RT-qPCR result showed that gga-miR-451 is significantly decreased in Exo-MG as compared with 
control group (Fig. 7C). Recently, we reported that gga-miR-451 decreased the inflammatory cytokine production, including TNF- $\alpha$ and IL-1 $\beta$ (16). We hypothesize that CP- $\bowtie$ cellular exosome-derived gga-miR451 can affect inflammatory cytokine levels in DF-1 cells. To test this hypothesis, DF-1 cells were separately incubated with Exo-MG, Exo-NC $(10 \mu \mathrm{g} / \mathrm{mL})$, transfected with miR-451 mimics (miR-451), or a nonspecific control siRNA (miR-451-NC). TNF- $\alpha$ and IL-1 $\beta$ protein levels were detected by ELISA at 36h post-treated. As shown in Figure 7D and E, the Exo-MG could notably increase TNF-a and IL-1 $\beta$ protein levels in DF-1 cells compared with Exo-NC. These results indicate that gga-miR-451 was selectively unloaded in MG-infection exosomes and may play a crucial role in the immune inflammatory process during MG infection.

\subsection{CP- $\$ cellular exosomederived gga-miR-451 regulates YWHAZ Expression in DF-1 cells}

YWHAZ is an established target gene of gga-miR-451. To confirm that exosomal gga-miR-451 can affect TNF- $\alpha$ and IL-1 $\beta$ expression through regulating YWHAZ, we separately cultured DF-1 cells with Exo-MG or Exo-NC derived from CP- $₫$ cells $(10 \mu \mathrm{g} / \mathrm{mL}$ ), transfected with miR-451 mimics (miR-451), or negative control (miR-451-NC). As expected, gga-miR-451 significantly decreased the mRNA and protein levels of YWHAZ as compared with NC, Exo-MG significantly increased the protein levels of YWHAZ as compared with Exo-NC, using RT-qPCR and Western blot (Figure 8 A and B). These data suggest that exosomes

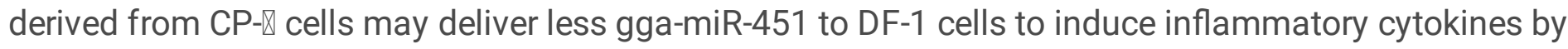
targeting YWHAZ.

\section{Discussion}

Inflammation, characterized by marked secretion of inflammatory cytokines, has the physiological purpose of restoring tissue homeostasis. Nevertheless, uncontrolled or unresolved inflammation can lead to tissue damage causing several diseases characterized by chronic inflammatory states (37).

Mycoplasma provokes rock-ribbed inflammation in organisms, resulting in the production of various proinflammatory cytokines in many different cell types (38). MG is well known as a common pathogen that can cause chronic respiratory inflammation in poultry worldwide. After MG-HS enters the chicken's body, it adheres to the respiratory tract mucous membrane and the epithelial cells through adhesin proteins (pMGA1.2), causing damage to the respiratory tract tissue. MG-HS then multiplies locally to induce lung cell degeneration, causing the cilia to stop moving and falling off, thereby infecting the host respiratory tract and establishing a chronic infection (39). After passing through the respiratory mucosal barrier, MG enters the blood circulation, spreading to tissues and organs throughout the body. Therefore, the damage to the cells of the respiratory tract and the resulting lesions are the leading link in the pathogenesis of this

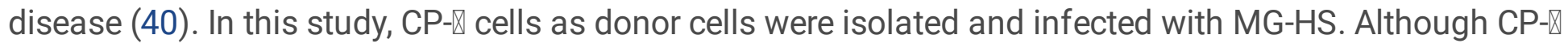
cells are not immune and inflammatory cells, there is increasing evidence that they play an important role in regulating lung immune defense $(24,41)$. 
Exosomes shuttling is a process with a wide range of important regulatory functions. They harbor a communicative function that involving an exchange of membranes or membrane proteins between exosomes and cells (42). Exosomes have been reported to play a crucial role in inflammation processes due to their capability to carry inflammatory modulators such as miRNA and proteins which can act on both neighboring and distal cells and affect cellular function (43). The special membrane structure of exosomes reinforces the stability of the miRNA and enhances its potential as a novel predictive marker in disease diagnosis. In addition, many functional experiments have demonstrated that exosomes affect lung injury and inflammation via exosomal miRNAs $(44,45)$. We sought to investigate the miRNA expression profiles of exosomes derived from MG-infected CP- $₫$ cells using Illumina HiSeq 2500 highthroughput sequencing (miRNA-seq) and the role of specific exosomal miRNA. The findings provide new insight into immunological variations during MG infection.

In this study, we identified $30(P<0.05$, fold-change $>2)$ significant $D E G$ s between the MG-infected and control group, including 9 up-regulated and 21 down-regulated, which can be chosen as the target miRNA to understand the possible molecular mechanism of exosomal miRNAs derived from MG-infected CP- $\mathbb{X}$ cells. The levels of urinary exosomal miR-193a were significantly higher in children with primary focal segmental glomerulosclerosis than those in children with minimal change disease (46). In Polymicrobial Sepsis, exosomal miR-223 contributes to Mesenchymal Stem Cell-Elicited Cardio protection (47). In our study, the expression of exosomal miR-193a and miR-223 was significantly changed in the exosomes

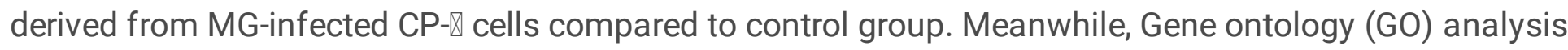
showed that the target genes of differentially expressed miRNAs (DEGs) mainly from a class of membrane-associated or organelle proteins ("membrane", "intracellular membrane-bounded organelle", "intracellular part", "cytoplasm") with small molecule binding and enzyme catalysis functions ("organic cyclic compound binding", "nucleic acid binding", "protein binding", "catalytic activity"). They are involved in the regulation of cellular process, metabolism, and protein modification ("cellular process", "metabolic process regulation of response to stimulus", "biological regulation"). The KEGG pathways is widely used to predict the gene function and genome information, which is helpful for us to study the metabolism function, genetic information processing, cellular processes and diseases (48). In this study, the DEGs were plotted to the reference pathways in KEGG. The most significant KEGG pathways were apoptosis, cell cycle, focal adhesion, tight junction, influenza A, Toll-like receptor signaling pathway, mTOR signaling pathway, Wnt signaling pathway, Cytokine-cytokine receptor interaction and MAPK signaling pathway. These pathways reflect the physiological processes of MG-infection and the potential regulatory mechanisms underlying them. Our previous data demonstrated that Toll-like receptor signaling pathway, Wnt signaling pathway, and MAPK signaling pathway play a crucial role in MG-HS infection and implicated in chronic inflammatory reaction $(9,36), \mathrm{DF}-1$ cells cycle and apoptosis are also modulated during MG-HS infection (16). Upregulation of gga-miR-451 significantly induced the MG-infected DF-1 cell-cycle arrest and promoted apoptosis (16). It has been well documented in the literature that miR100-5p-abundant exosomes derived from infrapatellar fat pad mesenchymal stem cells (MSCs) protect articular cartilage and ameliorate gait abnormalities via inhibition of mTOR in osteoarthritis (49). Together, the results indicate that exosomal miRNAs derived from MG-infected CP-II cells played a 
complex role in signaling pathways and regulating the inflammatory reactions, which provides the theoretical basis, direction for the prevention and treatment of Mycoplasma infection in poultry.

Our results revealed that the concentration of exosomes derived from MG-infected CP- $\$ cells was increased as non-infected cells (Fig. 1E, Table S3)," which was similar to the results of previous reports in other diseases $(35,50)$. Moreover, the presence of protease-rich exosomes in the airway secretions from subjects with cystic fibrosis lung disease colonized by gram-negative bacteria. LPS stimulation of airway epithelial cells also could lead to release of exosomes in human (51). Among the 8 validated exosomal miRNAs, 6 miRNAs were confirmed by RT-qPCR and deep sequencing to share similar trends in MGinfection and control groups. Although the validated miRNAs did not cover all the DEGs in the two libraries, the normalized profiling data and the RT-qPCR results indicated the reliability of our analysis results regarding the exosomal miRNAs distribution in the two groups. Interestingly, there are only 5 differentially expressed miRNAs (gga-miR-365-3p, gga-miR-449b-5p, gga-miR-133c-3p, gga-miR451, gga-miR-223) which are consistent between the results of this study and our previous results of miRNAs sequencing of MG-infected chicken lungs at 3 and 10 days post-infection (9). This study demonstrated that gga-miR-451 level was significantly increased in MG-infected CP- $\$ cells, which was shared by the results of our previous report in DF-1 cells (16). While the exosomal gga-miR-451 was significantly decreased in the exosomes derived from MG-infected CP- $\triangle$ cells compared to control group (Fig. 6A and 5).. These results suggest that gga-miR-451 were selected unloaded in exosomes derived from MG-infected CP- $₫$ cells. Under certain stimulating conditions, the epithelial cell-secreted exosomes are capable of causing or aggravating pulmonary inflammation. In this study, the Exo-MG could notably increase TNF-a and IL-1 $\beta$ protein levels in DF-1 cells compared to Exo-NC (Fig. 6D and E).. It is reported that exosomes, like vesicles released by adipose tissue of obese mice, are taken up by mononuclear cells inducing their differentiation into active macrophages with an increased release of TNF- $a$ and IL-6 (52). TNF- $a$ and IL- 6 increased via Rho associated coiled-coil-containing protein kinase 1 (ROCK1)-mediated pathways after treating alveolar macrophages with hyperoxia-induced, epithelial cell-derived exosomespacked caspase $-3(53,54)$. The blood microparticles treatment led to significant increases in myeloperoxidase (MPO), TNF- $a, \mathrm{IL}-1 \beta$, and IL-10 productions in bronchoalveolar lavage fluid and plasma (55). Our previous data showed that gga-miR-451 expression significantly decreased TNF- $a$ and IL-1 $\beta$ secretions in DF-1 cells (16). These were consistent with our results. A few papers have reported exosomal miRNA-451 has a diagnostic ability for diseases. For example, urinary exosomal miRNA-451 may hold prognostic value as an early and sensitive non-invasive indicator of renal disease (56). In nonsmall cell lung cancer, plasma exosomal miR-451a might serve as a reliable biomarker for prediction of recurrence and prognosis (57). It is reasonable to believe that gga-miR-451-desent exosomes derived from MG-infected CP- $₫$ cells increases inflammatory cytokine production, including TNF- $\alpha$ and IL-1 $\beta$.

Growing evidences have investigated YWHAZ protein (14-3-3ろ) in multiple systems and found that is a target gene and is negatively regulated by miR-451 in mammals and chickens $(16,58)$. We also found that gga-miR-451-desent exosomes can regulate the expression of YWHAZ protein in DF-1 cells (Fig. 7).

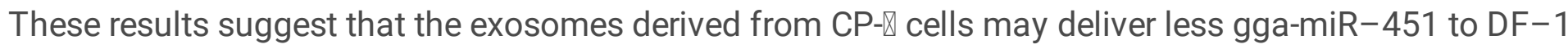
cells to induce inflammatory cytokines by targeting YWHAZ and increasing its expression. In addition to 
gga-miR-451, other exosomal gga-miRNAs may also regulate the immunity processes in MG infection. For example, exosomal miR-223 targets the transcription factor STAT5 and is implicated in inflammatory reactions in multiple sclerosis (59). The serum exosome derived microRNA-193 was reported to be a potential Alzheimer's Disease biomarker (60). However, the molecular mechanism of these exosomal miRNAs that shuttle and regulate chronic respiratory inflammation needs to be further studied.

\section{Conclusions}

The present study provides evidence to prove that the mode of intercellular communication between chicken cells is mediated by exosomes. About 30 exosome-microRNAs derived from CP-囚 were significantly changed following MG infection. The changed exosome-microRNAs target genes involved in cell cycle, Toll-like receptor signaling pathway and MAPK signaling pathway, etc. Additionally, exosomes from MG-infected CP- $₫$ cells alter the dynamics of the DF-1 cells, and may contribute to pathology of the MG infection via exosomal gga-miR-451 targeting YWHAZ involving in inflammation. These findings provide a new mechanism for the pathophysiology of MG infection and suggest exosomal gga-miR-451 as a new therapeutic target for the treatment of CRD.

\section{Abbreviations}

miRNAs: microRNAs; MG: Mycoplasma gallisepticum; CRD: chronic respiratory disease; CP-囚: chicken type $₫$ pneumocytes; DEGs: differentially expressed miRNAs; sRNA: small RNA; DF-1: chicken embryo fibroblast cell line; YWHAZ: tyrosine 3-monooxygenase/tryptophan 5-monooxygenase activation protein zeta; GAPDH: glyceraldehyde-3-phosphate dehydrogenase; IL: interleukin; DMEM: Dulbecco's modified Eagle's medium; TEM: transmission electron microscope; CCU: color-changing unit; NTA: nanoparticle tracking analysis; KEGG: Kyoto Encyclopedia of Genes and Genomes.

\section{Declarations}

\section{Acknowledgments}

The authors are grateful to Yanzhang Gong, Yanping Feng, Shijun Li and Zheya Sheng for their suggestions on writing this paper.

\section{Author Contributions}

Yabo Zhao and Yali Fu performed the experiments; Mengyun Zou and Yingfei Sun analyzed the data; Xun Yin and Lumeng Niu performed the total RNA isolation and cDNA preparation; Xiuli Peng conceived and designed the study and helped write the manuscript. All authors have read and approved the final manuscript for publication. 


\section{Funding}

This study was supported by the National Natural Science Foundation of China (Grant No. 31972681】 and the Fundamental Research Funds for the Central Universities (No. 2662017PY080).

\section{Availability of data and materials}

All data generated or analyzed during this study are included in this published article [and its supplementary information files].

\section{Ethics approval and consent to participate}

Our experimental protocols for chicken-embryo treatment were approved by the Institutional Animal Care and Use Committee of Huazhong Agricultural University. The procedures were carried out in accordance with the approved guidelines.

\section{Consent for publication}

Not applicable.

\section{Competing interests}

All authors listed declare that they have no competing interests.

\section{References}

1.Yuan B, Zou M, Zhao Y, Zhang K, Sun Y, Peng X. Up-Regulation of miR-130b-3p Activates the PTEN/PI3K/AKT/NF-kappaB Pathway to Defense against Mycoplasma gallisepticum (HS Strain) Infection of Chicken. Int J Mol Sci. 2018;19(8).

2.Machado LD, Santos FF, Togashi CK, Abreu DL, Pimentel JC, Sesti L, et al. Egg quality in laying hens exposed to Mycoplasma gallisepticum F-strain attenuated vaccine. Poult Sci. 2017;96(4):976-9.

3.Zhang N, Wu Y, Huang Z, Zhang C, Zhang L, Cai Q, et al. Relationship between danofloxacin PK/PD parameters and emergence and mechanism of resistance of Mycoplasma gallisepticum in In Vitro model. PLoS One. 2018;13(8):e0202070.

4.Elliott KEC, Branton SL, Evans JD, Peebles ED. Early post-hatch survival and humoral immune response of layer chickens when in ovo vaccinated with strain F Mycoplasma gallisepticum. Poult Sci. 2018;97(11):3860-9. 
5.Zhang H, Mehmood K, Jiang X, Li Z, Yao W, Zhang J, et al. Identification of differentially expressed MiRNAs profile in a thiram-induced tibial dyschondroplasia. Ecotoxicol Environ Saf. 2019;175:83-9.

6.Fabian MR, Sonenberg N, Filipowicz W. Regulation of mRNA translation and stability by microRNAs. Annu Rev Biochem. 2010;79:351-79.

7.MacDonagh L, Gray SG, Finn SP, Cuffe S, O'Byrne KJ, Barr MP. The emerging role of microRNAs in resistance to lung cancer treatments. Cancer Treat Rev. 2015;41(2):160-9.

8.Zhang L, Li J, Cui L, Shang J, Tian F, Wang R, et al. MicroRNA-30b promotes lipopolysaccharideinduced inflammatory injury and alleviates autophagy through JNK and NF-kappaB pathways in HK-2 cells. Biomed Pharmacother. 2018;101:842-51.

9.Zhao Y, Hou Y, Zhang K, Yuan B, Peng X. Identification of differentially expressed miRNAs through highthroughput sequencing in the chicken lung in response to Mycoplasma gallisepticum HS. Comparative biochemistry and physiology Part D, Genomics \& proteomics. 2017;22:146-56.

10.Zhang K, Han Y, Wang Z, Zhao Y, Fu Y, Peng X. gga-miR-146c Activates TLR6/MyD88/NF-kappaB Pathway through Targeting MMP16 to Prevent Mycoplasma Gallisepticum (HS Strain) Infection in Chickens. Cells. 2019;8(5).

11.Zhang K, Han Y, Zhao Y, Sun Y, Zou M, Fu Y, et al. Upregulated gga-miR-16-5p Inhibits the Proliferation Cycle and Promotes the Apoptosis of MG-Infected DF-1 Cells by Repressing PIK3R1Mediated the PI3K/Akt/NF-kappaB Pathway to Exert Anti-Inflammatory Effect. Int J Mol Sci. 2019;20(5).

12.Khordadmehr M, Jigari-Asl F, Ezzati H, Shahbazi R, Sadreddini S, Safaei S, et al. A comprehensive review on miR-451: A promising cancer biomarker with therapeutic potential. J Cell Physiol. 2019.

13.Rosenberger CM, Podyminogin RL, Navarro G, Zhao GW, Askovich PS, Weiss MJ, et al. miR-451 regulates dendritic cell cytokine responses to influenza infection. J Immunol. 2012;189(12):5965-75.

14.Fu C, Chen S, Cai N, Liu Z, Wang P, Zhao J. Potential neuroprotective effect of miR-451 against cerebral ischemia/reperfusion injury in stroke patients and a mouse model. World Neurosurg. 2019.

15.Feng L, Yang X, Liang S, Xu Q, Miller MR, Duan J, et al. Silica nanoparticles trigger the vascular endothelial dysfunction and prethrombotic state via miR-451 directly regulating the IL6R signaling pathway. Part Fibre Toxicol. 2019;16(1):16.

16.Zhao Y, Zhang K, Zou M, Sun Y, Peng X. gga-miR-451 Negatively Regulates Mycoplasma gallisepticum (HS Strain)-Induced Inflammatory Cytokine Production via Targeting YWHAZ. Int J Mol Sci. 2018;19(4).

17.Zhao Y, Fu Y, Sun Y, Zou M, Peng X. Transcriptional Regulation of gga-miR-451 by AhR:Arnt in Mycoplasma gallisepticum (HS Strain) Infection. Int J Mol Sci. 2019;20(12). 
18.Zhang Y, Hu YW, Zheng L, Wang Q. Characteristics and Roles of Exosomes in Cardiovascular Disease. DNA Cell Biol. 2017;36(3):202-11.

19.Mathivanan S, Ji H, Simpson RJ. Exosomes: extracellular organelles important in intercellular communication. J Proteomics. 2010;73(10):1907-20.

20.Sastre B, Canas JA, Rodrigo-Munoz JM, Del Pozo V. Novel Modulators of Asthma and Allergy: Exosomes and MicroRNAs. Front Immunol. 2017;8:826.

21.M HR, Bayraktar E, G KH, Abd-Ellah MF, Amero P, Chavez-Reyes A, et al. Exosomes: From Garbage Bins to Promising Therapeutic Targets. Int J Mol Sci. 2017;18(3).

22.Simhadri VR, Reiners KS, Hansen HP, Topolar D, Simhadri VL, Nohroudi K, et al. Dendritic cells release HLA-B-associated transcript-3 positive exosomes to regulate natural killer function. PLoS One. 2008;3(10):e3377.

23.Liu Z, Gan L, Zhang T, Ren Q, Sun C. Melatonin alleviates adipose inflammation through elevating alpha-ketoglutarate and diverting adipose-derived exosomes to macrophages in mice. J Pineal Res. 2018;64(1).

24.Zhang LY, Lv S, Wu SC, Guo X, Xia F, Hu XR, et al. Inhibitory effects of alpha-cyperone on adherence and invasion of avian pathogenic Escherichia coli 078 to chicken type II pneumocytes. Vet Immunol Immunopathol. 2014;159(1-2):50-7.

25.Bi D, Ji X. The isolation and identification of the mycoplasma gallisepticum. Acta Veterinaria et Zootechnica Sinica. 1988;1.

26.Bi D, Xu Q. Study on pathogenicity of HS strain Mycoplasma gallisepticum Chinese journal of animal and poultry infections diseases. 1997(5):24-6.

27.Calus D, Maes D, Vranckx K, Villareal I, Pasmans F, Haesebrouck F. Validation of ATP luminometry for rapid and accurate titration of Mycoplasma hyopneumoniae in Friis medium and a comparison with the color changing units assay. J Microbiol Methods. 2010;83(3):335-40.

28.Costa-Silva B, Aiello NM, Ocean AJ, Singh S, Zhang H, Thakur BK, et al. Pancreatic cancer exosomes initiate pre-metastatic niche formation in the liver. Nat Cell Biol. 2015;17(6):816-26.

29.Thery C, Amigorena S, Raposo G, Clayton A. Isolation and characterization of exosomes from cell culture supernatants and biological fluids. Curr Protoc Cell Biol. 2006;Chapter 3:Unit 322.

30.Nabet BY, Qiu Y, Shabason JE, Wu TJ, Yoon T, Kim BC, et al. Exosome RNA Unshielding Couples Stromal Activation to Pattern Recognition Receptor Signaling in Cancer. Cell. 2017;170(2):352-66 e13. 
31.Kamerkar S, LeBleu VS, Sugimoto H, Yang S, Ruivo CF, Melo SA, et al. Exosomes facilitate therapeutic targeting of oncogenic KRAS in pancreatic cancer. Nature. 2017;546(7659):498-503.

32.Li H, Durbin R. Fast and accurate short read alignment with Burrows-Wheeler transform. Bioinformatics. 2009;25(14):1754-60.

33.Rivals I, Personnaz L, Taing L, Potier MC. Enrichment or depletion of a GO category within a class of genes: which test? Bioinformatics. 2007;23(4):401-7.

34.Kanehisa M, Goto S. KEGG: kyoto encyclopedia of genes and genomes. Nucleic Acids Res. 2000;28(1):27-30.

35.Simeoli R, Montague K, Jones HR, Castaldi L, Chambers D, Kelleher JH, et al. Exosomal cargo including microRNA regulates sensory neuron to macrophage communication after nerve trauma. Nat Commun. 2017;8(1):1778.

36.Tian W, Zhao C, Hu Q, Sun J, Peng X. Roles of Toll-like receptors 2 and 6 in the inflammatory response to Mycoplasma gallisepticum infection in DF-1 cells and in chicken embryos. Dev Comp Immunol. 2016;59:39-47.

37.Takeda K, Akira S. Toll-like receptors in innate immunity. Int Immunol. 2005;17(1):1-14.

38.Beutler B. Inferences, questions and possibilities in Toll-like receptor signalling. Nature. 2004;430(6996):257-63.

39.Hu F, Zhao C, Bi D, Tian W, Chen J, Sun J, et al. Mycoplasma gallisepticum (HS strain) surface lipoprotein pMGA interacts with host apolipoprotein A-I during infection in chicken. Applied microbiology and biotechnology. 2016;100(3):1343-54.

40.Zhang HL, Chen ZM, Liu HMJZEKZZ. [Pathogenic mechanism of Mycoplasma pneumoniae infections]. 2016;54(2):94-7.

41.Zhang LY, Yi PF, Guo X, Wu SC, Fu YX, Zhang C, et al. Astragaloside IV Inhibits the Inflammatory Injury of Chicken Type II Pneumocytes Induced by Avian Pathogenic Escherichia coli. Inflammation. 2016;39(5):1660-9.

42.Duijvesz D, Luider T, Bangma CH, Urology GJJE. Exosomes as Biomarker Treasure Chests for Prostate Cancer. 2011;59(5):823-31.

43.Valadi H, Ekstrom K, Bossios A, Sjostrand M, Lee JJ, Lotvall JO. Exosome-mediated transfer of mRNAs and microRNAs is a novel mechanism of genetic exchange between cells. Nat Cell Biol. 2007;9(6):654-9.

44.Raposo G, Nijman HW, Stoorvogel W, Liejendekker R, Harding CV, Melief CJ, et al. B lymphocytes secrete antigen-presenting vesicles. J Exp Med. 1996;183(3):1161-72. 
45.Li ZL, Lv LL, Tang TT, Wang B, Feng Y, Zhou LT, et al. HIF-1 1alpha inducing exosomal microRNA-23a expression mediates the cross-talk between tubular epithelial cells and macrophages in tubulointerstitial inflammation. Kidney Int. 2019;95(2):388-404.

46. Huang Z, Zhang Y, Zhou J, Zhang Y. Urinary Exosomal miR-193a Can Be a Potential Biomarker for the Diagnosis of Primary Focal Segmental Glomerulosclerosis in Children. Biomed Res Int. 2017;2017:7298160.

47.Wang X, Gu H, Qin D, Yang L, Huang W, Essandoh K, et al. Exosomal miR-223 Contributes to Mesenchymal Stem Cell-Elicited Cardioprotection in Polymicrobial Sepsis. Sci Rep. 2015;5:13721.

48.Koh W, Sheng CT, Tan B, Lee QY, Kuznetsov V, Kiang LS, et al. Analysis of deep sequencing microRNA expression profile from human embryonic stem cells derived mesenchymal stem cells reveals possible role of let-7 microRNA family in downstream targeting of hepatic nuclear factor 4 alpha. BMC Genomics. 2010;11 Suppl 1:S6.

49.Wu J, Kuang L, Chen C, Yang J, Zeng WN, Li T, et al. miR-100-5p-abundant exosomes derived from infrapatellar fat pad MSCs protect articular cartilage and ameliorate gait abnormalities via inhibition of mTOR in osteoarthritis. Biomaterials. 2019;206:87-100.

50.Ren J, Zhou Q, Li H, Li J, Pang L, Su L, et al. Characterization of exosomal RNAs derived from human gastric cancer cells by deep sequencing. Tumour Biol. 2017;39(4):1010428317695012.

51.Szul T, Bratcher PE, Fraser KB, Kong M, Tirouvanziam R, Ingersoll S, et al. Toll-Like Receptor 4 Engagement Mediates Prolyl Endopeptidase Release from Airway Epithelia via Exosomes. Am J Respir Cell Mol Biol. 2016;54(3):359-69.

52.Deng ZB, Poliakov A, Hardy RW, Clements R, Liu C, Liu Y, et al. Adipose tissue exosome-like vesicles mediate activation of macrophage-induced insulin resistance. Diabetes. 2009;58(11):2498-505.

53.Lee H, Zhang D, Zhu Z, Dela Cruz CS, Jin YJSR. Epithelial cell-derived microvesicles activate macrophages and promote inflammation via microvesicle-containing microRNAs. 2016;6:35250.

54.Moon HG, Cao Y, Yang J, Lee JH, Choi HS, Jin Y. Lung epithelial cell-derived extracellular vesicles activate macrophage-mediated inflammatory responses via ROCK1 pathway. Cell Death Dis. 2015;6:e2016.

55.Li H, Meng X, Liang X, Gao Y, Cai S. Administration of microparticles from blood of the lipopolysaccharide-treated rats serves to induce pathologic changes of acute respiratory distress syndrome. Exp Biol Med (Maywood). 2015;240(12):1735-41.

56.Mohan A, Singh RS, Kumari M, Garg D, Upadhyay A, Ecelbarger CM, et al. Urinary Exosomal microRNA-451-5p Is a Potential Early Biomarker of Diabetic Nephropathy in Rats. PLoS One. 2016;11(4):e0154055. 
57.Kanaoka R, linuma H, Dejima $H$, Sakai T, Uehara $H$, Matsutani $N$, et al. Usefulness of Plasma Exosomal MicroRNA-451a as a Noninvasive Biomarker for Early Prediction of Recurrence and Prognosis of NonSmall Cell Lung Cancer. Oncology. 2018;94(5):311-23.

58.Bergamaschi A, Katzenellenbogen BS. Tamoxifen downregulation of miR-451 increases 14-3-3zeta and promotes breast cancer cell survival and endocrine resistance. Oncogene. 2012;31(1):39-47.

59.Ebrahimkhani S, Vafaee F, Young PE, Hur SSJ, Hawke S, Devenney E, et al. Exosomal microRNA signatures in multiple sclerosis reflect disease status. Sci Rep. 2017;7(1):14293.

60.Yang TT, Liu CG, Gao SC, Zhang Y, Wang PC. The Serum Exosome Derived MicroRNA-135a, -193b, and -384 Were Potential Alzheimer's Disease Biomarkers. Biomed Environ Sci. 2018;31(2):87-96.

\section{Table}

Table 1. List of significantly different exosomal miRNAs presented in the exosomes (Fold Change). 


\begin{tabular}{llll}
\hline sRNA & Up/Down & Log2 Fold Change & P-value \\
\hline gga-let-7d & down & -1.1638 & $1.12 \mathrm{E}-05$ \\
gga-miR-193a-3p & up & 1.3701 & $1.53 \mathrm{E}-05$ \\
\hline gga-miR-451 & down & -1.0392 & $4.19 \mathrm{E}-05$ \\
\hline gga-miR-133c-3p & down & -1.2484 & $5.16 \mathrm{E}-05$ \\
\hline gga-miR-33-5p & up & 1.1421 & $6.86 \mathrm{E}-05$ \\
\hline gga-miR-223 & down & -1.4015 & $9.18 \mathrm{E}-05$ \\
\hline gga-miR-460b-5p & up & 1.199 & 0.00011881 \\
\hline gga-miR-144-5p & down & -1.0368 & 0.00021744 \\
\hline gga-miR-1574-3p & down & -1.9138 & 0.0006816 \\
\hline gga-miR-365-3p & down & -1.2153 & 0.00120446 \\
\hline gga-miR-449c-5p & down & -1.5737 & 0.00228921 \\
\hline gga-miR-138-1-3p & down & -1.0125 & 0.00245147 \\
\hline gga-miR-3535 & down & -1.409 & 0.00301547 \\
\hline gga-miR-133b & down & -1.156 & 0.0037047 \\
\hline gga-miR-449c-3p & down & -1.9064 & 0.00477357 \\
\hline gga-miR-128-1-5p & down & -1.0753 & 0.00834198 \\
\hline gga-miR-1573 & down & -2.9042 & 0.00844519 \\
\hline gga-miR-202-5p & up & 3.0172 & 0.01188627 \\
\hline gga-miR-1553-5p & down & -2.0543 & 0.01246738 \\
\hline gga-miR-449b-5p & down & -1.0932 & 0.01649992 \\
\hline gga-miR-1784-5p & up & 2.3769 & 0.02353568 \\
\hline gga-miR-6550-3p & down & -2.0485 & 0.02378457 \\
\hline gga-miR-6555-3p & up & 1.1886 & 0.02466438 \\
\hline gga-miR-1648-5p & down & -5.4263 & 0.02542181 \\
\hline gga-miR-1783 & up & 4.985 & 0.02648076 \\
\hline gga-miR-199b & down & -1.7126 & 0.0286867 \\
\hline gga-miR-6696-3p & up & 1.3609 & 0.03085024 \\
\hline gga-miR-218-3p & up & 1.2401 & -1.1512 \\
\hline gga-miR-1788-5p & down & -2.4316 & \\
\hline gga-miR-1771 & down & & \\
\hline
\end{tabular}

\section{Figures}


A

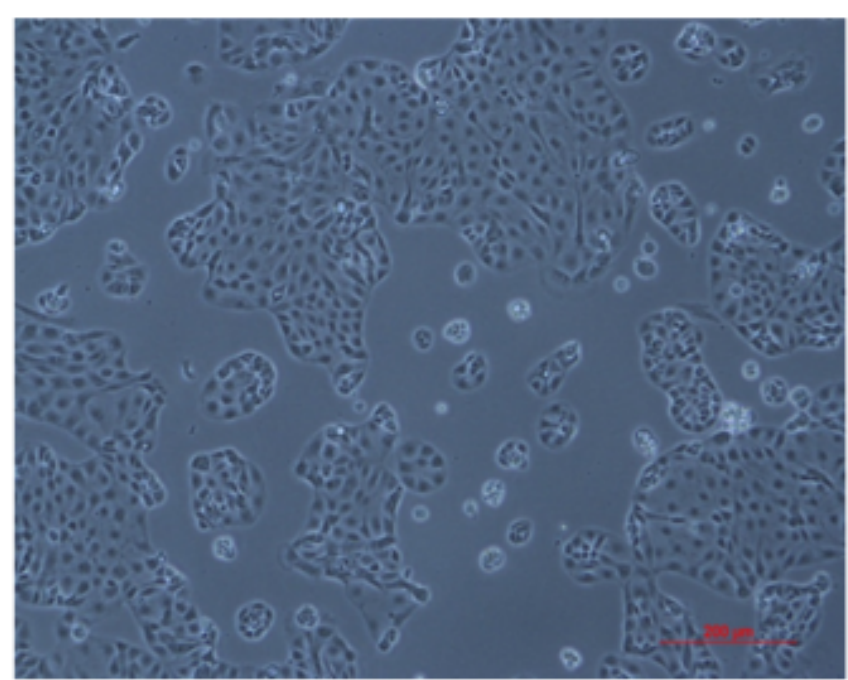

C

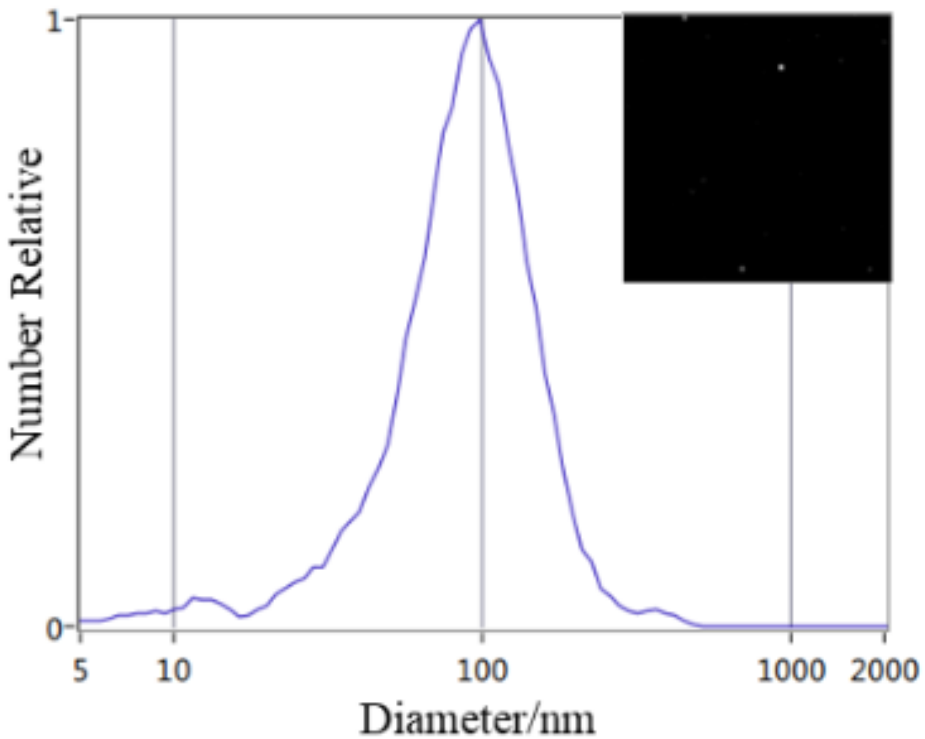

B

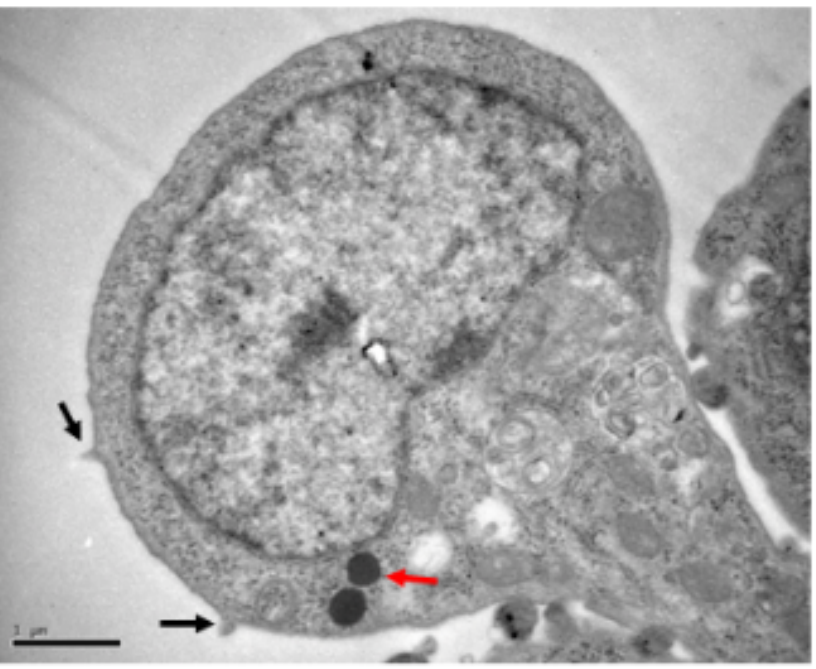

D

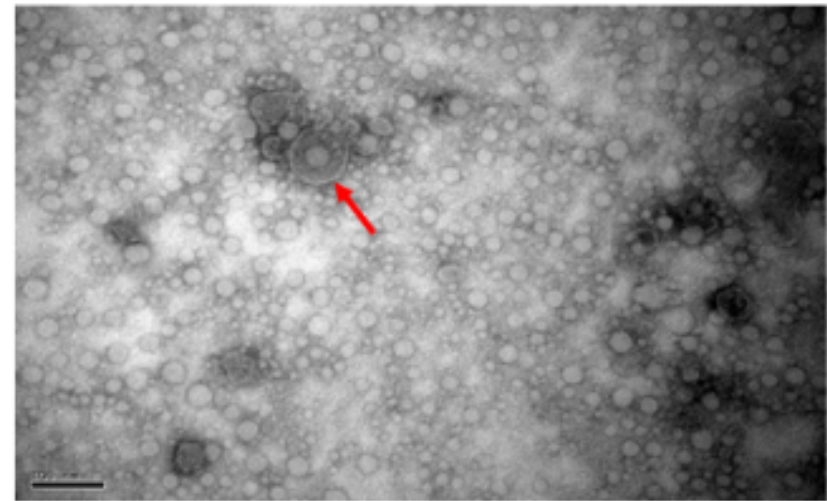

$\mathbf{E}$

MG-1 MG-2 NC-1 NC-2

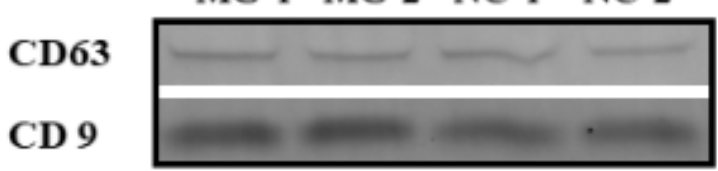

\section{Figure 1}

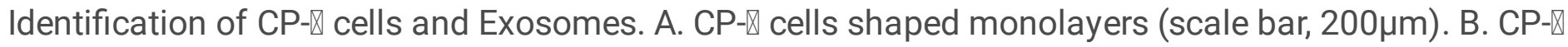
cells structure (Red arrow indicates the osmiophilic lamellar body, black arrow indicates microvilli; scale bar, $1 \mu \mathrm{m})$. C. Particle size distribution in exosome-enriched fractions. D. Electron micrograph showing whole-mount exosomes isolated from the supernatant fluid of cultured CP- $₫$ cells (Red arrow indicates exosomes; scale bar, 100nm). E. Western blot analysis showing exosome marker CD63, CD9. 

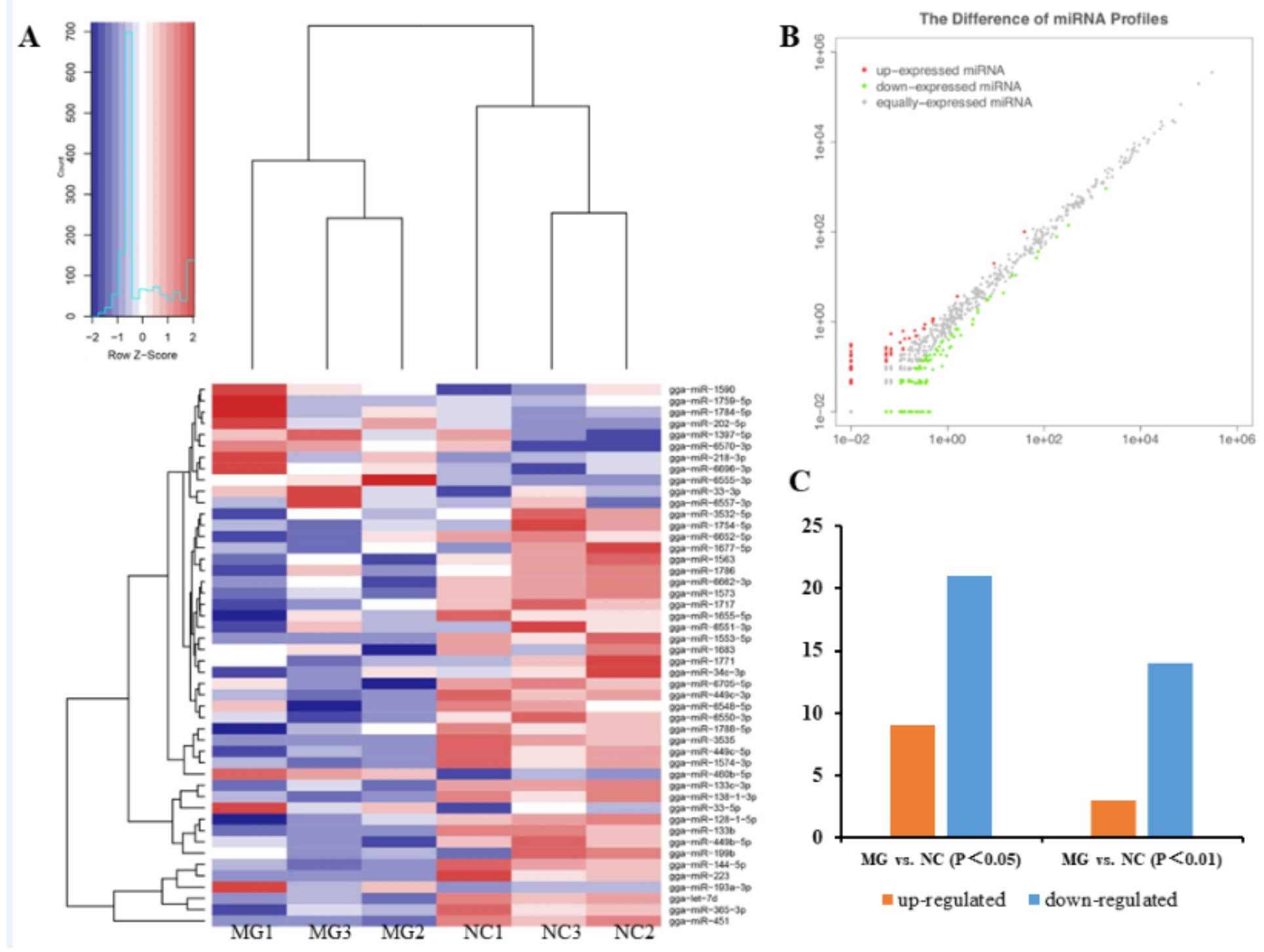

Figure 2

Cluster analysis of differentially expressed miRNAs. A. Heatmap of differentially expressed miRNAs between two groups (each row indicates a microRNA and each column represents one sample; the color scale illustrates the microRNA expression level). B. The difference of miRNAs profiles (each red point indicates up-expressed miRNA, each green indicates down-expressed miRNA and gray indicates equallyexpressed miRNA). C. Differentially expressed miRNAs. 


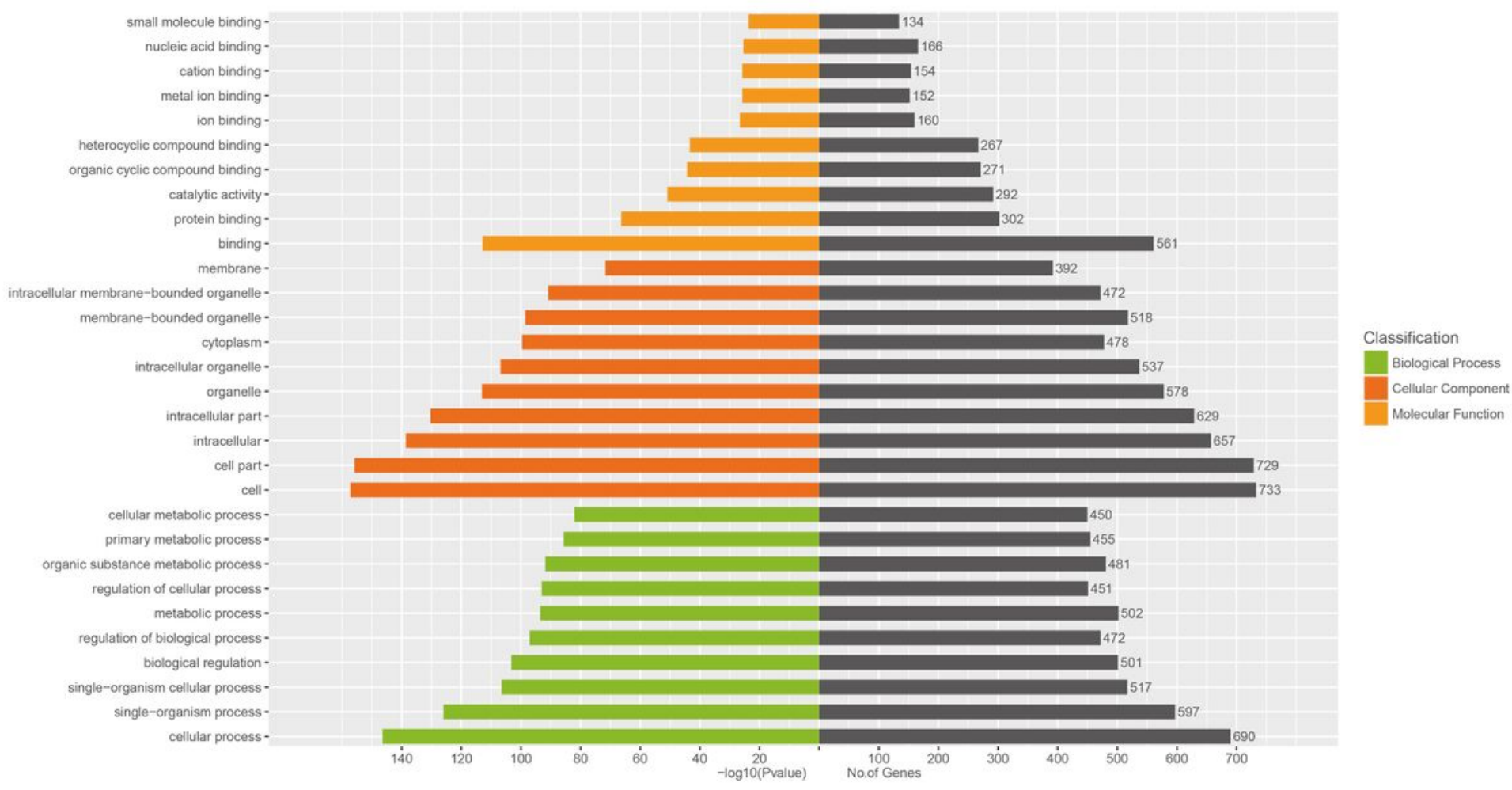

\section{Figure 3}

Enriched GO terms of different expression exosomal miRNAs. (blue represents the biological process; red represents cellular component and orange indicates molecular function). The $\mathrm{Y}$-axis shows gene functions, and the X-axis corresponds to the percent of miRNAs and number of samples.

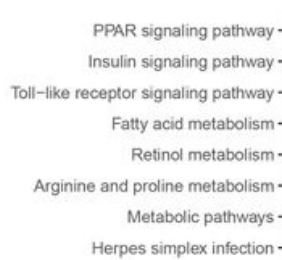

Herpes simplex infection-

AGE-RAGE signaling pathway in diabetic complications Influenza A -

Protein processing in endoplasmic reticulum -

Ubiquitin mediated proteolysis Calcium signaling pathway MAPK signaling pathway FoxO signaling pathwayVEGF signaling pathway mTOR signaling pathway Wnt signaling pathway Cytokine-cytokine receptor interaction Cell adhesion molecules (CAMs) ErbB signaling pathway Lysosome-

Adherens junction Oocyte meiosis -

Regulation of actin cytoskeletonCell cycle Endocytosis Tight junction Focal adhesion Apoptosis -

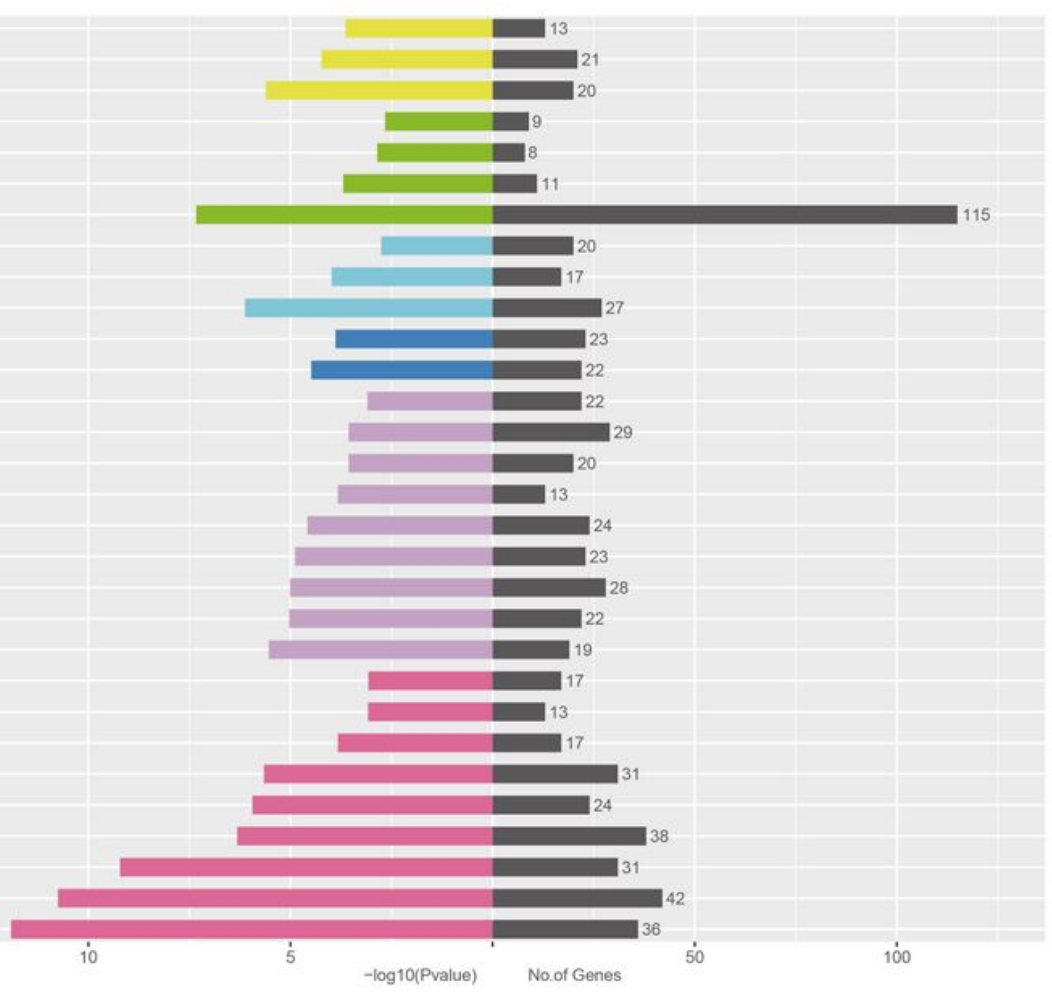

Classification

Cellular Processes Environmental Information Processing Genetic Information Processing Human Diseases Metabolism Organismal Systems

\section{Figure 4}


KEGG enrichment analysis of differentially expressed miRNAs in chicken type II pneumocytes-derived exosomes (the most enriched top 30 pathway terms - pink, cellular processes - purple, environmental information processing - blue, genetic information processing - light blue, human diseases - green, metabolism - yellow, organismal systems). The horizontal axis denoted the -log10 (P-value) and the number of miRNAs, while the vertical axis indicated the pathway name. The right gray histogram indicates the numbers of samples in each cluster.

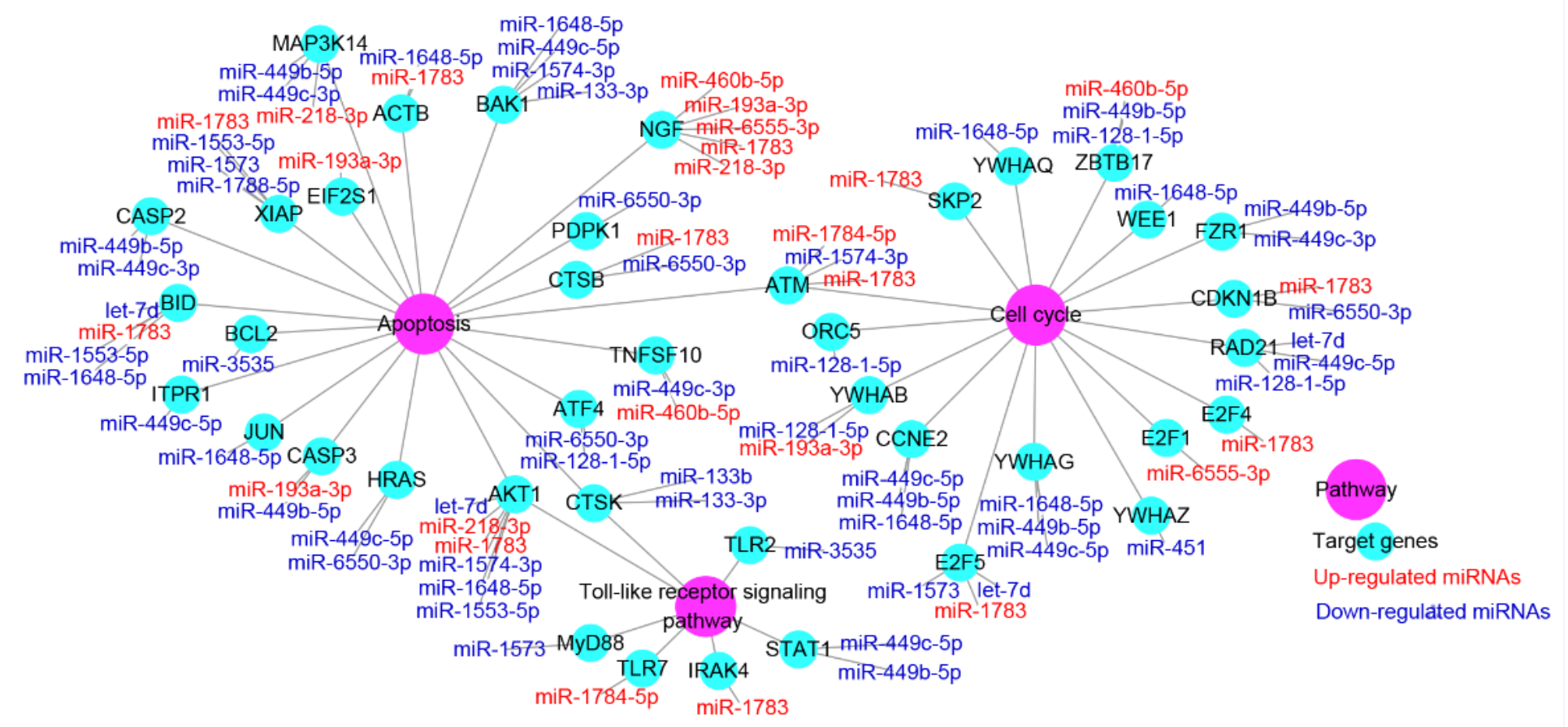

\section{Figure 5}

Network of miRNA-target pathways involved in cell cycle, apoptosis and Toll-like receptor signaling pathway for differential expressed miRNAs. The pink represents the pathways, green represents the genes, the red represents the up-regulated miRNAs and the blue represents the down-regulated miRNAs. 


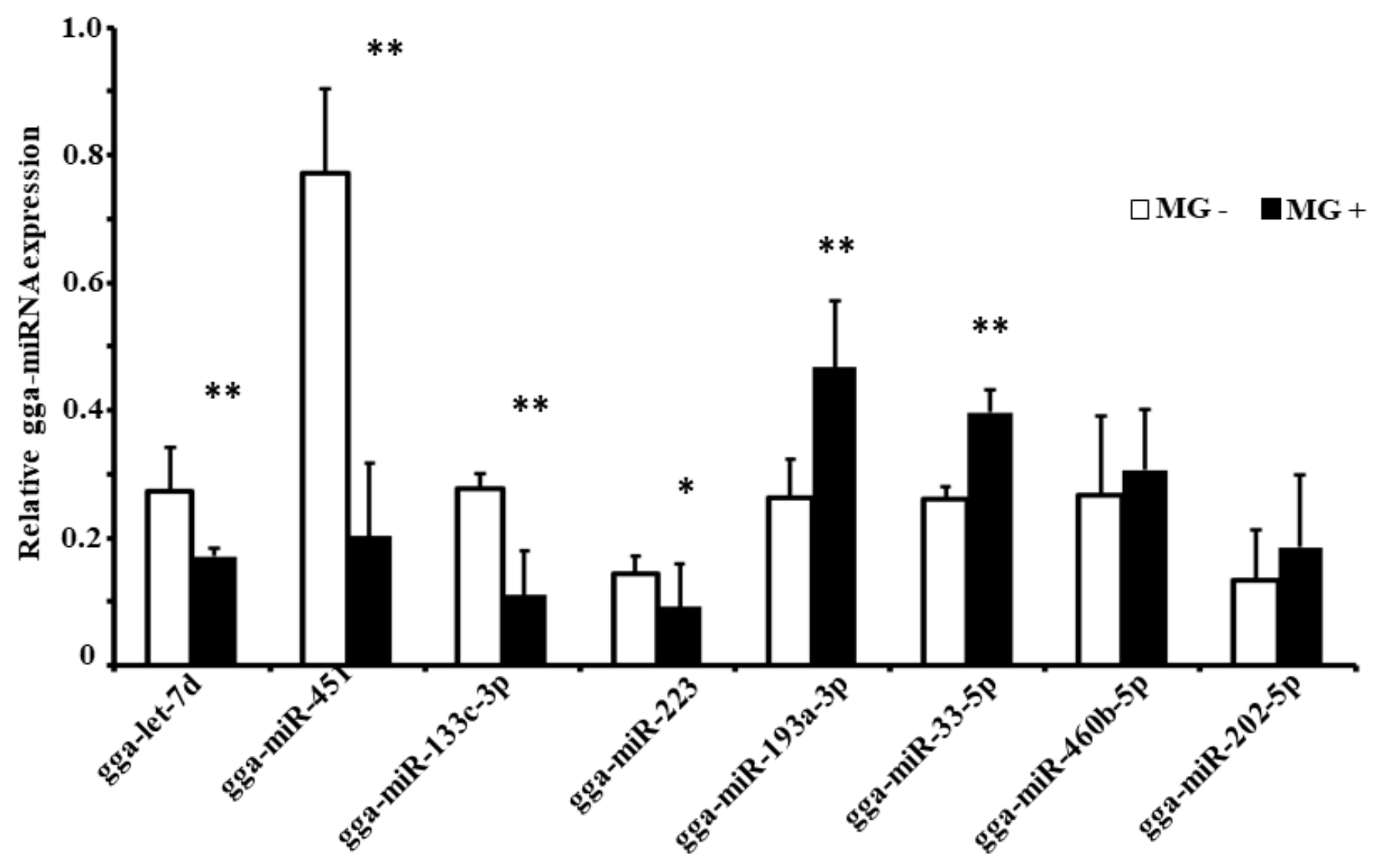

Figure 6

The validation of the selected exosome-microRNA expression profile approach by using RT-qPCR. 5s-RNA was used as the internal control, Data represent means \pm SDs of three independent experiments (Twotailed Student t-test, ${ }^{*} \mathrm{p}<0.01,{ }^{\star} \mathrm{p}<0.05$ ) 
A

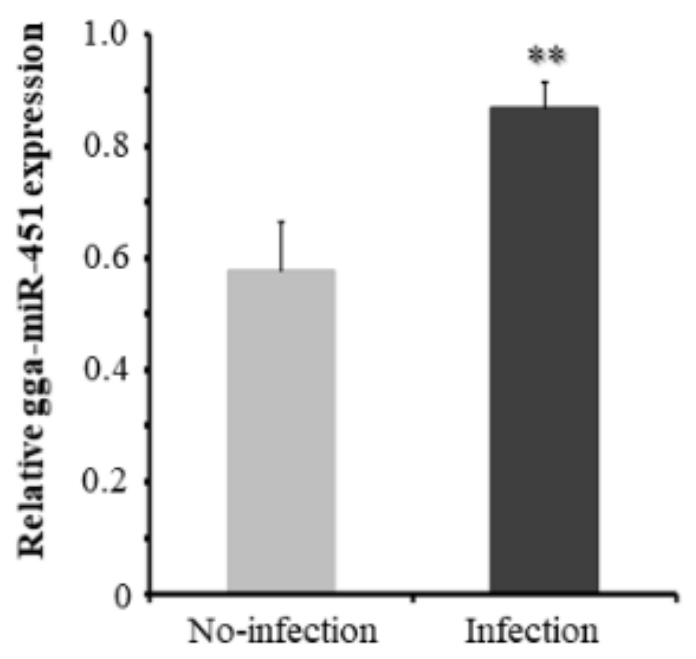

$\mathrm{D}$

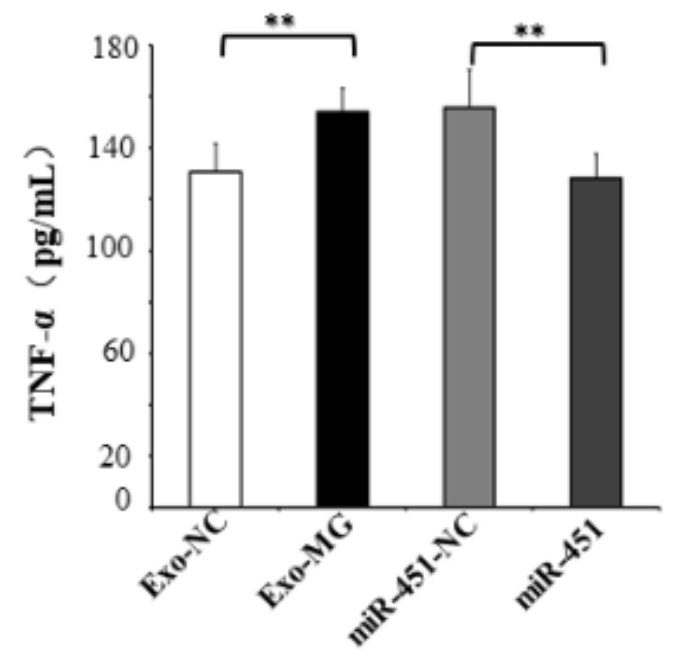

$\mathrm{C}$

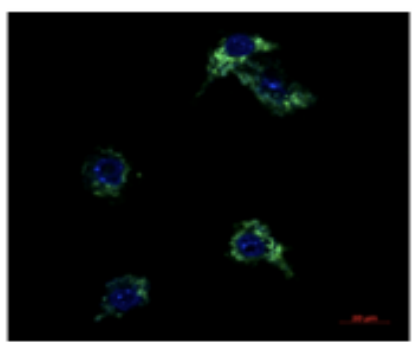

Exosome/DAPI
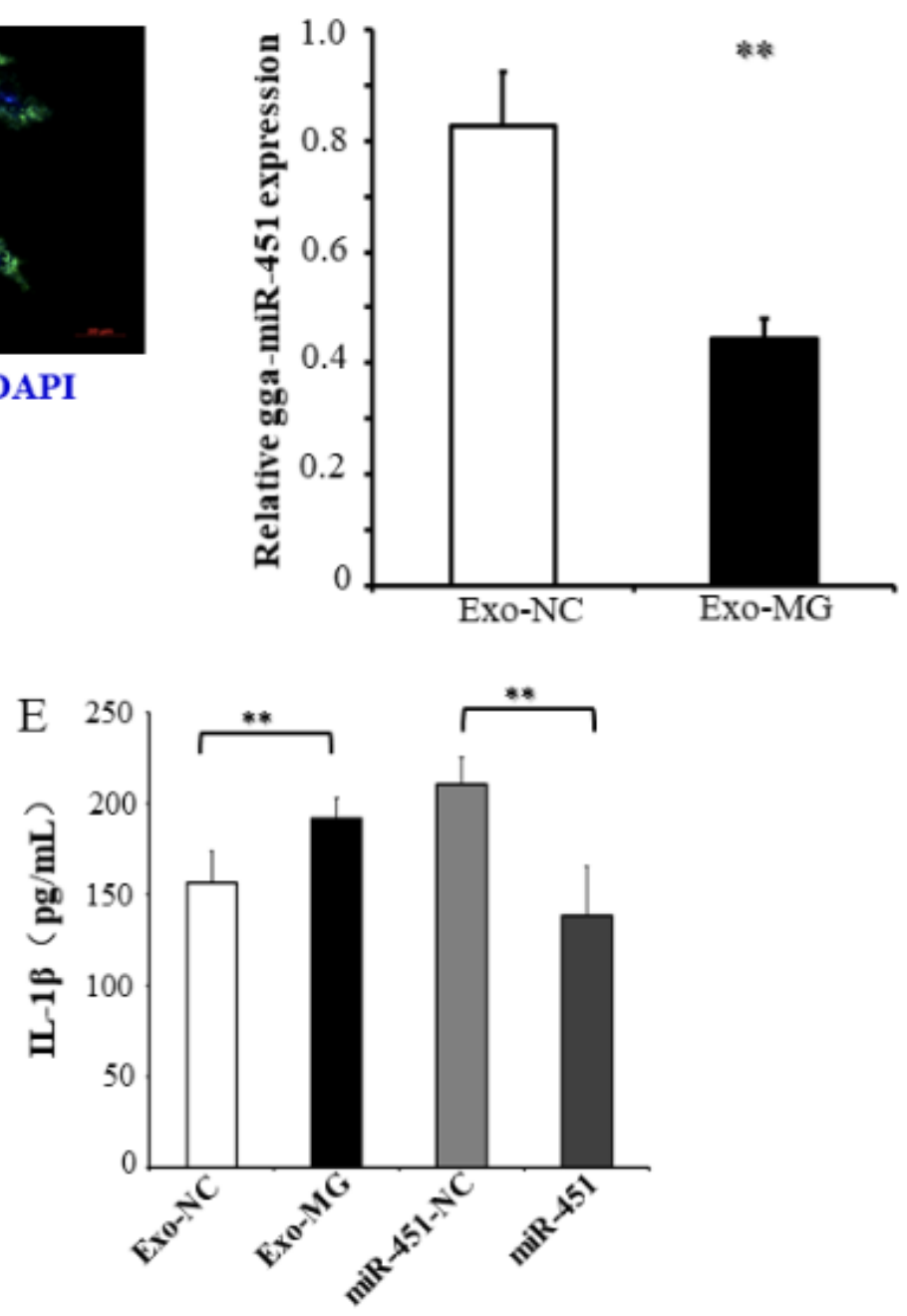

Figure 7

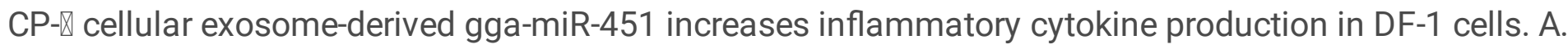
Relative gga-miR-451 expression in MG-HS infected CP- $₫$ cells. B. CP- $\Downarrow$ cells-derived exosomes were labeled with PKH67 (green), incubated with DF-1 cells, and then observed by confocal microscopy, cell nuclei (blue), exosomes (green). C. Relative gga-miR-451 expression in DF-1 cells cultured with CP- $\triangle$ cellsderived exosomes (Exo-MG or Exo-NC). 5s-RNA was used as the internal control. D-E. The inflammatory cytokine level of TNF- $\alpha$ and IL-1 $\beta$ in DF-1 cells at $48 \mathrm{~h}$ post-treatment. Exo-MG and Exo-NC represent exosomes isolated from MG-infected and non-infected CP- $\$ cells, separately. Data represents three independent experiments and are presented as the means \pm SDs (Two-tailed Student's t-test, ${ }^{\star *} p<0.01$ ). 


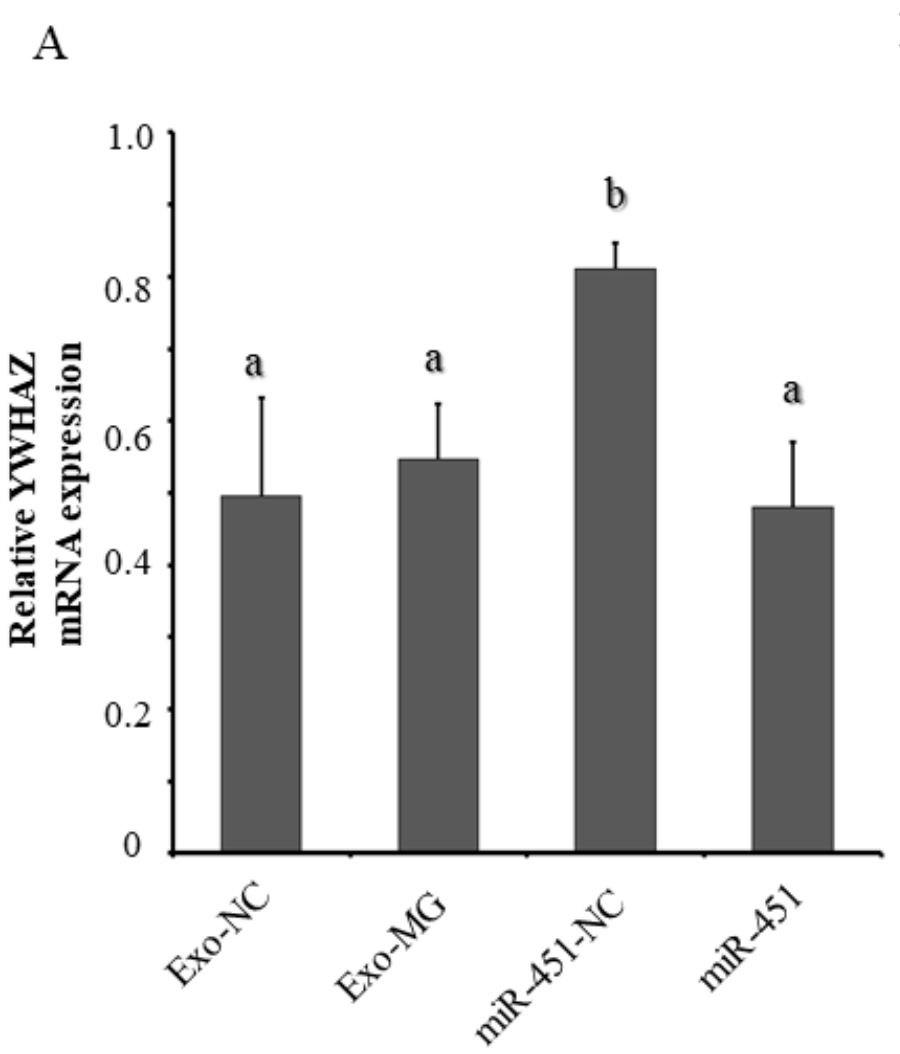

B
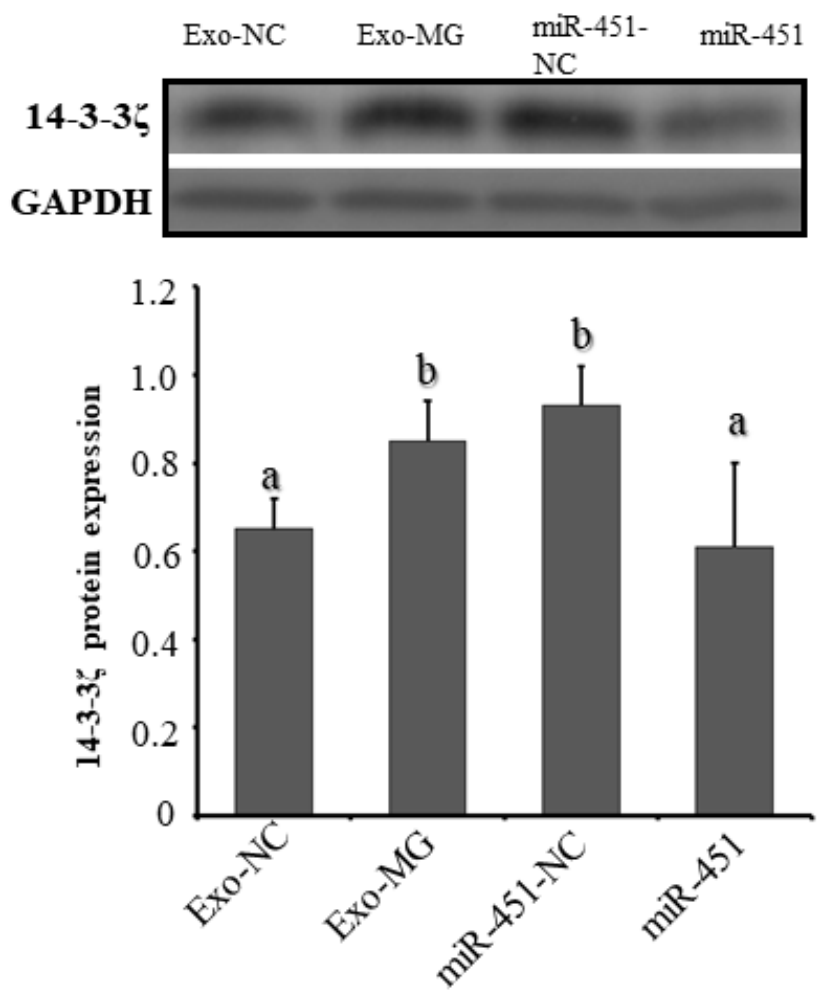

Figure 8

CP- $\rrbracket$ cellular exosome-derived gga-miR-451 regulates YWHAZ Expression. A. YWHAZ mRNA expression was detected by RT-qPCR. B. Western blot analyzes of 14-3-3 3 expression level in different treatment groups. GAPDH was used as the internal control. Exo-MG and Exo-NC represent exosomes isolated from MG-infected and non-infected CP- $\varangle$ cells, respectively. Data represent three independent experiments and are presented as the mean \pm SDs (Two-tailed Student's t-test, different lowercase letters represent $\mathrm{P}<$ $0.01)$.

\section{Supplementary Files}

This is a list of supplementary files associated with this preprint. Click to download.

- SupplementaryTable3.docx

- Rawimagefiles.pdf

- SupplementaryTable2.docx

- SupplementaryTable4.docx

- SupplementaryTable1.docx 